\title{
The incorporation of soil-structure interaction into performance-based design
}

\author{
J. Avilés ${ }^{1} \&$ L. E. Pérez-Rocha ${ }^{2}$ \\ ${ }^{1}$ Instituto Mexicano de Tecnología del Agua, Mexico \\ ${ }^{2}$ Centro de Investigación Sísmica, FJBS, Mexico
}

\begin{abstract}
A performance-based design is aimed at controlling the structural damage relying on-precise estimations of proper response parameters. This is possible if more accurate analyses are carried out, including all potentially important factors involved in the structural behaviour, as is the case of soil-structure interaction. This paper presents a simplified procedure for practical damage analysis of structures elastically supported, with potential application to performance-based design. The damage model proposed here is based on maximum displacement and dissipated energy under monotonic loading, with the effects of cyclic behaviour and load reversals being estimated using a modified Park-Ang index. Analyses are made for a single elastoplastic structure with embedded foundation in a stratum over rigid bedrock. This is done by using an analogy with an equivalent fixed-base oscillator defined by the coupled period and damping of the system and its global ductility. Selected numerical results are presented in terms of dimensionless parameters, the significance of soil-structure interaction in the structural performance is elucidated and the adequacy of the approach presented is examined.
\end{abstract}

Keywords: damage model, equivalent oscillator, performance-based design, soilstructure interaction, strength and damage spectra.

\section{Introduction}

It is widely recognised that the performance-based design requires more accurate analyses, including all potential important factors involved in the structural behaviour. Only in this way, the prediction of the expected level of structural damage associated with a given level of earthquake can be improved. 
One of these factors is soil-structure interaction (SSI). There is still controversy regarding the role of SSI in the seismic performance of structures placed on soft soil. For elastic systems, the effect of foundation flexibility is to enlarge the structure period $[1,2]$. As a consequence, depending on the resonant period in the response spectrum, one may expect either an increase or a decrease of structural response. The response will undergo additional changes due to the increase or decrease in damping by wave radiation [1,2]. A recent study by Avilés and Pérez-Rocha [3] has revealed that the peak response of inelastic systems is affected less by SSI compared to that of elastic systems. In terms of strength and displacement demands, the SSI effects are found to be beneficial for structures with fundamental period longer that the prevailing period of the site, but detrimental if the structure period is shorter than the site period.

Although the SSI effects are less important for inelastic than for elastic systems, they may have significant influence on the structural performance under severe earthquakes [4]. The performance-based design procedures, however, are still inadequate to account for the effects of SSI. A number of damage models have been developed for evaluation of seismic performance, but usually assuming the structure as rigidly supported. Different damage indices have been proposed for this purpose, an overview of which can be found in Bozorgnia and Bertero [5]. The most recognised of them are functions of the maximum and cumulative demands of plastic deformation, quantities that are generally determined at the exclusion of SSI.

The structural performance depends not only on the maximum displacement demand, but also on the cumulative damage resulting from low-cycle fatigue [6]. In practical damage models, it is very difficult to consider the whole history of inelastic excursions. So, the use of dissipated hysteretic energy has been accepted as the response parameter best correlated with the cumulative damage. Perhaps, the most recognized damage model based on maximum displacement and dissipated energy is the one proposed by Park and Ang [7]. Besides other deficiencies [5], the Park-Ang index does not give correct results if the structure remains elastic and when the ultimate displacement capacity under monotonically increasing load is reached.

In this paper, a simplified procedure to incorporate SSI into performance-based design is presented. To achieve this, a consistent damage index that is zero for incipient damage and unity for potential collapse is applied. It is implemented on an equivalent fixed-base oscillator recently proposed by the authors [3], which is characterised by the coupled period and damping of the system and its global ductility. Two types of SSI spectra are presented, namely, strength spectra for constant damage and damage spectra for specified strength.

\section{Simplified reference model}

The soil-structure system under investigation is shown in fig. 1. It consists of an elastoplastic one-story structure placed on a rigid mat that is supported on springs and dampers and excited by input motions. The springs connected to the base account for the horizontal and rocking flexibilities of the foundation, whereas the 
dampers account for both material and radiation damping in the soil. The dynamic stiffnesses $\left(K_{h}, C_{h}\right.$ and $\left.K_{r}, C_{r}\right)$ and input motions $\left(U_{0}\right.$ and $\left.\Phi_{0}\right)$ are quantities depending on the exciting frequency, foundation geometry and soil characteristics. The input motions also depend on the nature of the earthquake excitation. Here, these quantities are computed for a circular foundation embedded in a stratum over rigid bedrock under vertically propagating shear waves, using the approximate formulas derived by Kausel et al. [8].

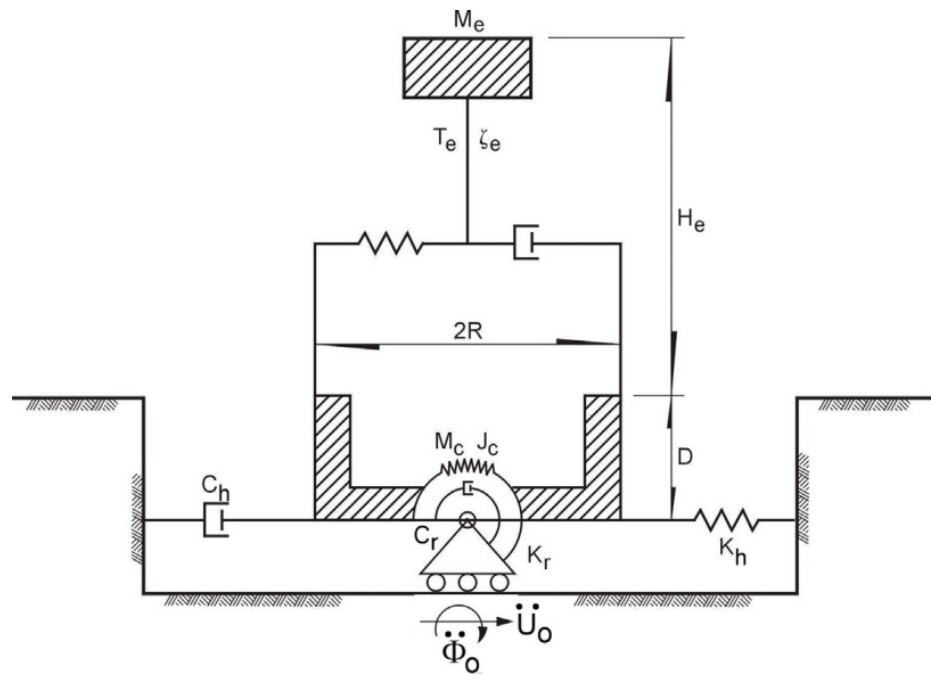

Figure 1: $\quad$ Model used to investigate the significance of SSI in performancebased design.

The structure is characterized by the height $H_{e}$ and mass $M_{e}$; its natural period and damping ratio for the elastic and fixed-base conditions are $T_{e}$ and $\zeta_{e}=0.05$. The foundation is defined by the radius $R$, depth of embedment $D$, mass $M_{c}$ and mass moment of inertia $J_{c}$ about a horizontal centroidal axis. Throughout the paper, it will be assumed that $M_{e} / \rho_{s} \pi R^{2} H_{e}=0.15, \rho_{s}$ being the mass density of the soil, $M_{c} / M_{e}=0.25$ and $J_{c} / M_{e}\left(H_{e}+D\right)^{2}=0.05$.

\subsection{Equivalent fixed-base oscillator}

Design concepts for performance-based earthquake engineering are generally developed by reference to a single oscillator. Thus, the most promising approach for incorporating SSI seems to be the introduction of an equivalent fixedbase oscillator. The procedures developed for replacing the actual flexible-base structure by an equivalent fixed-base oscillator usually do not account for the ductile capacity of the structure [1,2]. Recently, however, Avilés and Pérez-Rocha [3] have shown that the actual structure with natural period $T_{e}$, damping ratio $\zeta_{e}$ and ductility factor $\mu_{u}$ can be properly replaced by an equivalent oscillator whose 
properties are given by the coupled period and damping of the system, $\tilde{T}_{e}$ and $\tilde{\zeta}_{e}$, and its global ductility $\tilde{\mu}_{u}$. If the foundation's mass and moment of inertia are disregarded, the relationships of the system's period and damping, $\tilde{T}_{e}$ and $\tilde{\zeta}_{e}$, to those of the structure alone, $T_{e}$ and $\zeta_{e}$, are given by [3]

$$
\begin{gathered}
\tilde{T}_{e}=\left(T_{e}^{2}+T_{h}^{2}+T_{r}^{2}\right)^{1 / 2}, \\
\tilde{\zeta}_{e}=\zeta_{e} \frac{T_{e}^{3}}{\tilde{T}_{e}^{3}}+\frac{\zeta_{h}}{1+2 \zeta_{h}^{2}} \frac{T_{h}^{2}}{\tilde{T}_{e}^{2}}+\frac{\zeta_{r}}{1+2 \zeta_{r}^{2}} \frac{T_{r}^{2}}{\tilde{T}_{e}^{2}}
\end{gathered}
$$

where $T_{h}=2 \pi\left(M_{e} / K_{h}\right)^{1 / 2}$ and $T_{r}=2 \pi\left[M_{e}\left(H_{e}+D\right)^{2} / K\right]^{1 / 2}$ are the natural periods associated with rigid-body translation and rocking of the structure, whereas $\zeta_{h}=\pi C_{h} / \tilde{T}_{e} K_{h}$ and $\zeta_{r}=\pi C_{r} / \tilde{T}_{e} K_{r}$ are the damping ratios of the soil for the horizontal and rocking modes of the foundation.

To have an equivalent oscillator with the same strength and plastic deformation capacity as the actual structure, it is required that [3]

$$
\tilde{\mu}_{u}=1+\left(\mu_{u}-1\right) \frac{T_{e}^{2}}{\tilde{T}_{e}^{2}}
$$

This expression represents the global ductility of the system and implicitly assumes that the translation and rocking of the foundation are the same in both yielding and ultimate conditions, which holds when the soil remains elastic and the structure behaves elastoplastically.

It is interesting to note that, with this approach, the yield strength of the equivalent oscillator $\left(\tilde{V}_{y}\right)$ for the global ductility $\tilde{\mu}_{u}$ proves to be the same as that required by the actual structure $\left(V_{y}\right)$ for the allowable ductility $\mu_{u}$, when both systems are subjected to the same base excitation. In contrast, there is a difference between the maximum displacements of the two systems, given by [3]

$$
U_{u}=\frac{T_{e}^{2}}{\tilde{T}_{e}^{2}} \frac{\mu_{u}}{\tilde{\mu}_{u}} \tilde{U}_{u},
$$

where $U_{u}$ and $\tilde{U}_{u}$ are the ultimate displacements of the actual structure and equivalent oscillator, respectively. The reason for this is that the displacement of the equivalent oscillator involves both the relative displacement of the structure as well as the contribution by the translation and rocking of the foundation. In other words, both systems would experience the same plastic deformation, but different total displacement. 


\subsection{Dissipated hysteretic energy}

The plastic energy dissipated by the structure under earthquake excitation is computed as the total area enclosed by the hysteresis loops developed during the time-history response. For a general elastoplastic cycle, we have that

$$
E_{H}^{c}=V_{y}\left(U_{m}-U_{y}\right),
$$

where $U_{m}$ is the inelastic displacement demand and $U_{y}=V_{y} / K_{e}$ the yield displacement, $K_{e}$ being the initial stiffness of the structure. In view of what has been discussed, the inelastic displacement of the actual structure should be obtained by subtracting the rigid-body motion due the foundation flexibility from the inelastic displacement of the equivalent oscillator. As the yield displacements of both systems are $U_{y}=U_{u} / \mu_{u}$ and $\tilde{U}_{y}=\tilde{U}_{u} / \tilde{\mu}_{u}$, it follows from eqn (4) that $U_{y}=\left(T_{e}^{2} / \tilde{T}_{e}^{2}\right) \tilde{U}_{y}$. The difference between $\tilde{U}_{y}$ and $U_{y}$ represents the rigid-body motion due to the foundation flexibility. As a result, the inelastic displacement demand can be written as

$$
U_{m}=\tilde{U}_{m}-\left(1-T_{e}^{2} / \tilde{T}_{e}^{2}\right) \tilde{U}_{y}
$$

This expression is a generalized representation of eqn (4). It is apparent from eqn (6) that $U_{m}=\tilde{U}_{m}$ when there is no SSI, i.e. $\tilde{T}_{e}=T_{e}$. Next, it will be shown that the equivalent oscillator has the same capacity of plastic energy dissipation as the actual structure.

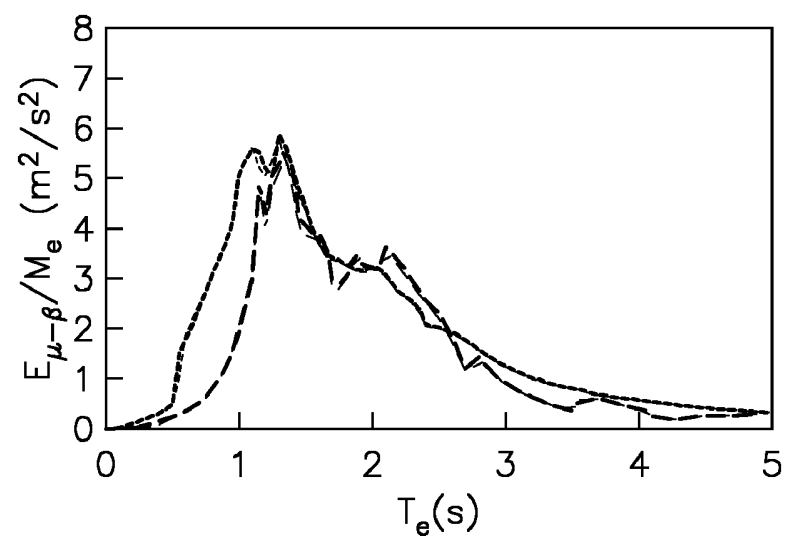

Figure 2: $\quad$ Hysteretic energy spectra per unit mass for $\mu \mathrm{u}=2$ (dashed line) and 4 (dotted line); comparison of the exact solution for the actual structure (thick line) with the approximate solution for the equivalent oscillator (thin line). 
As free-field control motion, the 1985 Michoacan earthquake was used; this was recorded at site SCT, representative of the soft zone in Mexico City. The dominant period of this site is $T_{s}=2 \mathrm{~s}$ and its damping and Poisson's ratios are $\zeta_{s}$ $=0.05$ and $v_{s}=0.45$, respectively. To reduce the number of examples, only one system configuration was considered, given by: $H_{e} / R=3$, for the slenderness ratio of the structure; $D / R=0.5$, for the embedment ratio of the foundation; and $H_{s} / R=3$, for the normalized depth of the stratum.

Hysteretic energy spectra per unit mass $\left(E_{\mu-\beta} / M_{\mathrm{e}}\right)$ against the fixed-base period are exhibited in fig. 2 for $\mu_{u}=2$ and 4 . As can be seen, the energy demands calculated for the actual structure are well predicted by using the equivalent oscillator; it is not easy to detect the difference within the scale of drawings. This satisfactory agreement confirms the efficiency of the equivalent oscillator to readily compute hysteretic energy spectra with SSI.
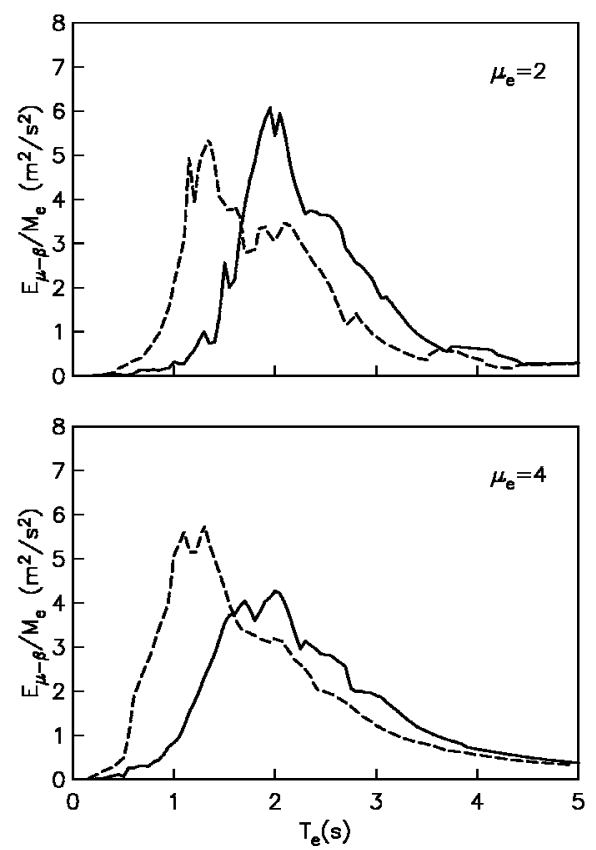

Figure 3: Hysteretic energy spectra per unit mass with (dashed line) and without (solid line) SSI.

The exact results of fig. 2 are now compared in fig. 3 with those corresponding to the fixed-base case. As has been observed with strength and displacement demands [3], the SSI effects can result in large increments or reductions of the energy demands, with respect to the fixed-base values, depending on the period ratio of the structure and site. In view of the period lengthening of structure, the hysteretic energy spectra with SSI tend to shift toward the left of those without SSI. In terms of the hysteretic energy dissipation, it is noted that SSI may affect the structural performance either adversely for $T_{e}<T_{s}$ or positively for $T_{e}>T_{s}$. 


\section{Consistent damage index}

In conventional design procedures only collapse- and damage-prevention limit states are usually considered. A more rational design procedure should permit the designer to evaluate other structural performance states, which are associated with different damage limit states. This can be realized by introducing an appropriate damage index into the non-liner analysis of the structure.

In the words of Bozorgnia and Bertero [5], a well-defined damage index for a single oscillator is a normalized quantity that will be zero if the structure remains elastic and will be unity if there is a potential of structural collapse. With this in mind, a consistent damage index was introduced by making a reasonable adjustment to the commonly used Park-Ang index, resulting in [9]

$$
D I=\alpha \frac{U_{m}-U_{y}}{U_{u}-U_{y}}+\beta \frac{E_{H}^{m}}{E_{H}^{u}}=\frac{\alpha\left(\mu_{m}-1\right)+\beta E_{H}^{n}}{\mu_{u}-1}
$$

where $U_{m}$ is the maximum displacement demand during earthquake excitation and $\mu_{m}=U_{m} / U_{y}$ the corresponding ductility demand, whereas $U_{u}$ is the ultimate displacement capacity under monotonic loading and $\mu_{u}=U_{u} / U_{y}$ the corresponding ductility capacity. Besides, $E_{H}^{m}$ is the hysteretic energy demand during earthquake excitation and $E_{H}^{u}=V_{v}\left(U_{u}-U_{y}\right)$ the hysteretic energy capacity under monotonic loading; $E_{H}^{n}=E_{H}^{m} / V_{y} U_{y}$ is the so-called normalized hysteretic energy. The coefficients $\alpha$ and $\beta$ control the strength deterioration in terms of the maximum displacement and dissipated energy, respectively. They depend on the characteristics of both the structural system and earthquake excitation, and should be determined on an experimental basis by imposing the condition $\alpha+\beta=1$. The values adopted here are $\alpha=0.85$ and $\beta=0.15$, and are used only to illustrate the implementation of damage models in analyses of SSI.

The value of $D I$ will be zero if $U_{m} \leq U_{y}$, implying elastic behavior and therefore no significant damage is expected. Also, under monotonically increasing load, the value of $D I$ will be one if $U_{m}=U_{u}$, implying potential failure. For the failure condition, $D I=1$, eqn (7) leads to

$$
\mu_{m}=\frac{\mu_{u}-\beta\left(1+E_{H}^{n}\right)}{\alpha}
$$

Notice that, for monotonic loading, $E_{H}^{n}=\mu_{u}-1$ and consequently $\mu_{m}=\mu_{u}$. As the energy dissipation capacity under cyclic loading is generally larger than that under monotonic loading, it follows from eqn (8) that $\mu_{m}<\mu_{\mu}$. This means that the monotonic ductility capacity is reduced by the effect of low-cycle fatigue. Indeed, eqn (8) defines what Fajfar [6] has called equivalent ductility factor to take into account the cumulative damage due to cyclic load reversals in the inelastic range. 


\section{SSI spectra}

As anticipated, two types of SSI spectra are presented: strength spectra for constant damage and damage spectra for specified strength. Strength spectra are constructed by iteration on the yield resistance until the $\mu_{m}$ and $E_{H}^{n}$ demands satisfy eqn (7), for given values of damage index corresponding to desired performance levels. Normalised strength spectra $\left(V_{y} / M_{e} g\right)$ against the fixed- base period are displayed in fig. 4 , for $\mu_{u}=4$ and a constant value of damage index, $D I$ $=1$. Results are given with and without regard to SSI and, in each case, spectra for constant damage are compared with those for constant ductility.
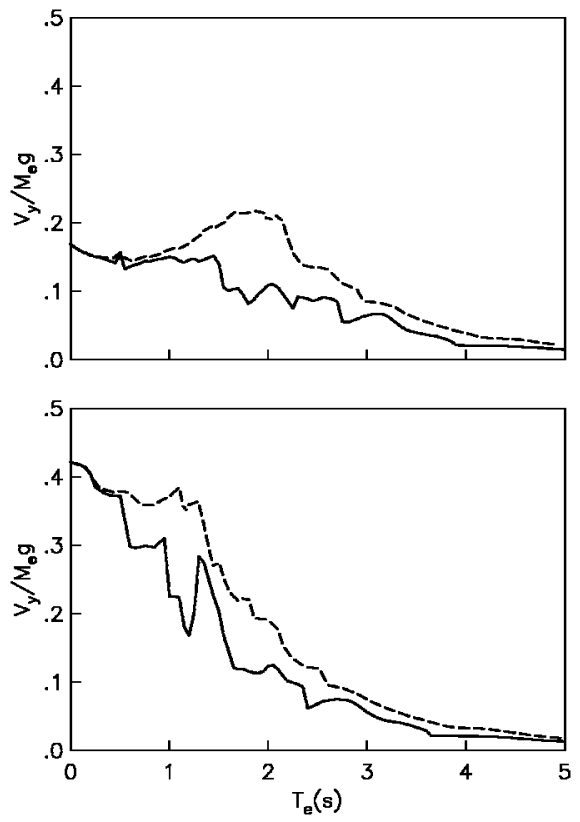

Figure 4: $\quad$ Normalised strength spectra for constant damage DI $=1$ (dashed line) and constant ductility $\mu \mathrm{u}=4$ (solid line), with (bottom) and without (top) SSI.

It can be seen that larger strengths are required when the structural damage is considered, especially around the site period for the fixed-base case. For flexible foundation, this occurs at $T_{e}<T_{s}$ due to the period lengthening of the structure. Irrespective of the foundation flexibility, the strengths required for very short and long periods of vibration are independent of the structural damage. For other natural periods, there are no theoretical indications regarding their values.

Damage spectra are constructed by proper combination of the $\mu_{m}$ and $E_{H}^{n}$ demands according to eqn (7), for specified yield strengths. Calculations were carried out for three values of the base-shear coefficient: $V_{y} / M_{e} g=0.1,0.2$ and 0.4 . 
The corresponding damage index spectra $\left(I D_{\mu-\beta}\right)$ are depicted in fig. 5. In general, the structural damage undergoes large increments for $T_{e}<T_{s}$ and slight reductions for $T_{e}>T_{s}$, with respect to the fixed-base values. It is seen that structures with $T_{e}$ $>T_{s}$ are well protected against collapse if $V_{y} / M_{e} g \geq 0.2$. Otherwise, the effect of low-cycle fatigue may produce collapse, especially when SSI is significant. It may be noticed that these results were derived for mid-rise structures, since $H_{e} / R=3$. Accordingly, they are not realistic over the whole range of natural periods, because such structures normally fall in the medium-period spectral region.
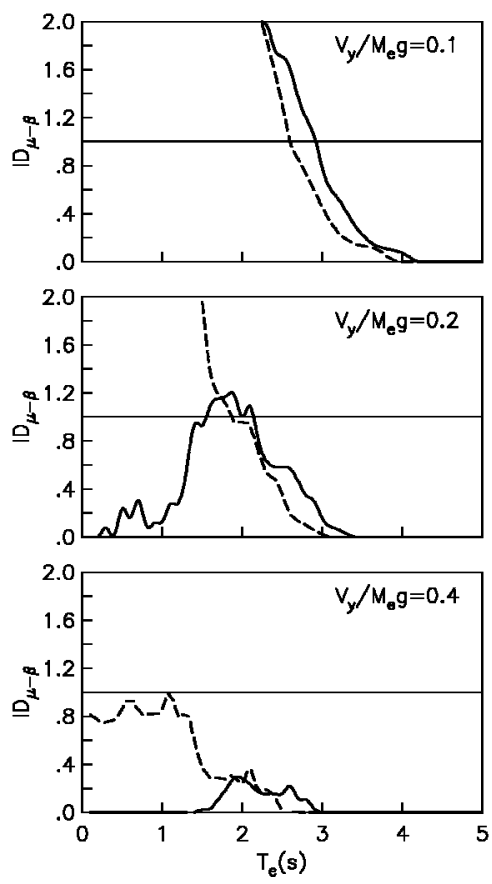

Figure 5: Damage index spectra for different base-shear coefficients, with (dashed line) and without (solid line) SSI.

\section{Concluding remarks}

The formulation of a simplified energy-based approach for damage analysis of structures with flexible foundation has been presented. A consistent damage index that is zero for incipient damage and unity for potential collapse was introduced. The concepts on inelastic response developed in practice for fixedbase structures were enlarged to account for SSI. This was done by using an analogy with an equivalent fixed-base oscillator defined by the coupled period and damping of the system and its global ductility. The equivalent oscillator was shown to have the same strength and energy dissipation capacity as the actual structure. Both constant damage spectra as well as constant strength spectra were 
evaluated with and without regard to SSI. It was found that the SSI effects may result in large increments or reductions of the spectral ordinates, with respect to the fixed-base values, depending on the period ratio of the structure and site.

\section{References}

[1] Jennings, P.C. \& Bielak, J., Dynamics of building-soil interaction. Bulletin of the Seismological Society of America, 63, pp. 9-48, 1973.

[2] Veletsos, A.S. \& Meek, J.W., Dynamic behaviour of building-foundation systems. Earthquake Engineering and Structural Dynamics, 3, pp. 121-138, 1973.

[3] Avilés, J. \& Pérez-Rocha, L.E., Soil-structure interaction in yielding systems. Earthquake Engineering and Structural Dynamics, 32, pp. 1749-1771, 2003.

[4] Reséndiz, D. \& Roesset, J.M., Soil-structure interaction in Mexico City during the 1985 earthquake. Proc. of the Int. Conf. on the 1985 Mexico Earthquakes, Factors Involved and Lessons Learned, ASCE, New York, 1986.

[5] Bozorgnia, Y. \& Bertero, V.V., Damage spectra: Characteristics and applications to seismic risk reduction. Journal of Structural Engineering, $A S C E$, 129, pp. 1330-1340, 2003.

[6] Fajfar, P., Equivalent ductility factors, taking into account low-cycle fatigue. Earthquake Engineering and Structural Dynamics, 21, pp. 837-848, 1992.

[7] Park, Y.J. \& Ang, A.H-S., Mechanistic seismic damage model for reinforced concrete. Journal of Structural Engineering, ASCE, 111, pp. 722-739, 1985.

[8] Kausel, E., Whitman, R.V., Morray, J.P. \& Elsabee, F., The spring method for embedded foundations. Nuclear Engineering and Design, 48, pp. 377-392, 1978.

[9] Avilés, J. \& Pérez-Rocha, L.E., Aspectos de interacción suelo-estructura en el diseño por desempeño, Proc. 7th Simposio Nacional de Ingeniería Sísmica, Mexico, 2002. 